OPEN ACCESS

Edited by:

Dong-Hua Yang,

St. John's University, United States

Reviewed by:

Samer Daher

Rutgers University, Newark,

United States

Sirisha Kondle,

Wayne State University, United States

*Correspondence:

Yu Yang

yangyu13836125585@163.com

${ }^{\dagger}$ These authors have contributed equally to this work

Specialty section: This article was submitted to

Gastrointestinal Cancers,

a section of the journal

Frontiers in Oncology

Received: 27 May 2021

Accepted: 12 August 2021

Published: 02 September 2021

Citation:

Bai Y, Ding M, LU D, Li Y, Yao S, Wang L, Li H, Cui G, Li X, Sun $X$ and Yang $Y$ (2021) Long Noncoding RNA

AC007639. 1 Promotes the

Pathogenesis and Progression of

Hepatocellular Carcinoma Through Inhibiting Apoptosis and Stimulating

Chemotherapeutic Resistance.

Front. Oncol. 11:715541.

doi: 10.3389/fonc.2021.715541

\section{Long Noncoding RNA AC007639.1 Promotes the Pathogenesis and Progression of Hepatocellular Carcinoma Through Inhibiting Apoptosis and Stimulating Chemotherapeutic Resistance}

\author{
Yun Bai ${ }^{1+}$, Meijuan Ding ${ }^{2 \dagger}$, Dan Lu ${ }^{1}$, Yiwen $\mathrm{Li}^{1}$, Shuai Yao ${ }^{3}$, Lei Wang ${ }^{4}$, Hui $\mathrm{Li}^{1}$, \\ Guanghua Cui ${ }^{1}$, Xue $L^{1}{ }^{1}$, Xiaoke Sun ${ }^{1}$ and $Y u$ Yang $^{1 *}$
}

\footnotetext{
${ }^{1}$ Department of Oncology, Second Affiliated Hospital of Harbin Medical University, Harbin, China, ${ }^{2}$ Department of Oncology, Harbin Medical University Cancer Hospital, Harbin, China, ${ }^{3}$ Department of Technology, Harbin Nachuan Bio-Science Technology Co., Ltd., Harbin, China, ${ }^{4}$ Department of Internal Medicine, Second Hospital of Heilongjiang Province, Harbin, China
}

Background: Hepatocellular carcinoma (HCC) is known for its poor prognosis. Long noncoding RNAs (IncRNAs) are critical in the pathogenesis of various types of cancers. We tried to explore the role of IncRNA in the development of HCC.

Methods: We identified the role of IncRNA AC007639.1 in the pathogenesis of HCC through bioinformatics and biological experiments in HepG2, Hep3B, and SMMC-7721 cells as well as the nude mice xenograft model.

Results: We found that IncRNA AC007639.1 was overexpressed in hepatocellular carcinoma. Knocking down of IncRNA AC007639.1 by specific siRNAs or shRNAs promoted cancer cell death. The growth of mouse xenograft tumor created using IncRNA AC007639.1 deficient HepG2 cells was significantly slowed down. Furthermore, the knockdown of IncRNA AC007639.1 in HCC cells led to the increased expression of p53 and decreased expression of angiopoietin-like 4.

Conclusion: LncRNA AC007639.1 was involved in the pathogenesis and progression of hepatocellular carcinoma by inhibition of apoptosis and increasing HCC resistance to chemotherapy.

Keywords: long noncoding RNA, hepatocellular carcinoma, bioinformatics, p53, chemotherapy

\section{INTRODUCTION}

The American Cancer Society estimates that there will be about 42,230 new cases of liver cancer, with 30,230 deaths due to liver cancer in the United States in 2021, representing a more than tripled incidence and more than doubled mortality since 1980. Worldwide, people diagnosed with liver cancer exceed 800,000 and more than 700,000 deaths are due to liver cancer each year, making liver 
cancer a leading cause of cancer-related deaths (1). One of the main causes of liver cancer, hepatitis B virus infection, is even more deleterious in leading to fulminant hepatitis (2-5), and the virus can be passed to the newborns without proper control of HBV level and HBV vaccination (6). Therefore, the mechanisms of tumorigenesis and factors affecting the prognosis of hepatocellular carcinoma (HCC) deserve more attention.

Long noncoding RNAs (lncRNAs), by definition, are not translated into proteins (7). Increasing evidence has shown that $\operatorname{lncRNAs}$ are involved in the pathogenesis and progression of different types of cancers $(8,9)$. Many of the lncRNAs are promising markers for the diagnosis and prognosis of liver cancer (10).

In this study, the role of IncRNA AC007639.1 in the pathogenesis and prognosis of HCC was explored by bioinformatics analysis and biological experiments using three different liver cancer cell lines. We found that lncRNA AC007639.1 knockdown led to increased cancer cell death. Importantly, lncRNA AC007639.1 could inhibit the p53 signaling pathway and increase the expression of Angiopoietinlike 4 (ANGPTL4) leading to inhibition of apoptosis and increasing of HCC resistance to chemotherapy.

\section{MATERIALS AND METHODS}

\section{Bioinformatic Analysis}

Transcriptome sequencing data including mRNA and lncRNA were generated by $\mathrm{R}$ package in Liver Hepatocellular Carcinoma Project of The Cancer Genome Atlas (TCGA-LIHC) dataset $(n=420)$ using TCGA biolinks (https://bioconductor.org/packages/release/data/ experiment/vignettes/TCGAbiolinksGUI.data/inst/doc/vignettes. html), and SRP069212 ( $\mathrm{n}=355)$ using Gene Expression Profiling Interactive Analysis (GEPIA; http://gepia.cancer-pku.cn/). Differential mRNA abundance was analyzed using DESeq2 (11). Genes with reads $>5$ were included in the final quantitative and statistical analysis. Heatmaps and volcano plots were prepared using the $\mathrm{R}$ package. Normalized gene expression levels were analyzed by Gene Set Variation Analysis (GSVA). Survival analysis was done by $\mathrm{R}$ package survival. Cox proportional hazard $(\mathrm{PH})$ model was constructed by $\mathrm{R}$ package (survminer). The best-scanned cutoff points had the most significant split (log-rank test).

\section{Cell Culture}

Human HCC cell lines HepG2 (RRID : CVCL_ 0027), SMMC7721 (RRID : CVCL_ 0534), and Hep3B (RRID : CVCL_ 0326), were obtained from the Shanghai Zhong Qiao Xin Zhou Biothechnology Co., Ltd., (Shanghai, China). The cells were confirmed to have no mycoplasma contamination using Mycoplasma Detection Kit (R\&D Systems China Co., Ltd., Shanghai, China). Dulbecco's modified Eagle's medium (DMEM, Gibco, Thermo Fisher Scientific, Inc.) was used for cell culture, with 10\% fetal bovine serum (FBS, Gibco), 2 mM Lglutamine (Gibco), $10 \mathrm{mM}$ HEPES (Gibco), $1 \mathrm{mM}$ pyruvate sodium (Gibco), and $100 \mathrm{U} / \mathrm{ml}$ penicillin with $100 \mu \mathrm{g} / \mathrm{ml}$ streptomycin (Gibco). Cells were grown at $37^{\circ} \mathrm{C}$ with $5 \% \mathrm{CO}_{2}$.

\section{Construction of Stable Knockdown of Lnc RNA AC007639.1 in HepG2 and Hep3B Cells}

Cells with stable lnc RNA AC007639.1-knockdown, ANGPTL4knockdown, or control HepG2-LNC-NC cells were created using shRNA 5'- GGUGAGUGCAUGUAGUCAUTT -3', 5'- AGAA CAGCAGGAUCCAGCAACUCUU -3', or scramble control sequence 5'- UUCUCCGAACGUGUCACGUTT -3'. shRNA oligos were cloned into a LV3(H1/GFP\&Puro) vector (map shown in Figure S1), respectively. The corresponding plasmids were packaged using lentivirus (Shanghai GenePharma, China). Puromycin ( $5 \mu \mathrm{g} / \mathrm{ml}$ final concentration, Sigma, St. Louis, MO) was used to select HCC cells carrying the transfected shRNA.

\section{siRNAs Transfection Into HCC Cells}

Specific short interfering RNAs (siRNAs) or control siRNA to knock down lnc RNA AC007639.1 were purchased from the Shanghai GenePharma (Shanghai, China). 30nM of siRNAs (Table S1) were used for transfection in the HCC cells using XtremeGENE reagent (Roche Applied Science, Shanghai, China).

\section{RNA Extraction and Quantitative RT-PCR}

For RNA extraction, cells or tissues were homogenized with TRIzol reagent (Thermo Fisher Scientific, Inc.). RNAs were purified and quantified using the NanoDrop 2000 Spectrophotometer (Thermo Fisher Scientific, Inc.). RNAs were reversely transcribed by the RT reagent Kit (Nachuan Bio-Tech Co., Binzhou, China). qRT-PCR experiments were done in $10 \mu \mathrm{L}$ total volume, which contained $1 \mathrm{x}$ SYBR Green Master mix (Nachuan Bio-Tech Co., China), cDNA (10 ng), and primers (75 $\mathrm{nM}$ of forward and $75 \mathrm{nM}$ reverse primers, Table S1) in an Exicycle 96 Real-Time Quantitative Thermal Block (Bioneer, China), with initial incubation at $95^{\circ} \mathrm{C}(10 \mathrm{~min}), 40$ cycles at $95^{\circ} \mathrm{C}(15 \mathrm{~s})$ and $60^{\circ} \mathrm{C}(1 \mathrm{~min})$. qRT-PCR experiments were triplicated, the averages of which were normalized (by $\beta$-actin), and the relative expression of AC007639.1 was calculated using the $2^{-\Delta \mathrm{CCt}}$ method.

\section{Cell Count Kit-8 Assay}

Cell proliferation was determined using the CCK-8 assay kit (DOJINDO, Japan). Briefly, cells ( 1 x 10\% /well) were grown in 96well plates. At the same time of each day $(10 \mathrm{am})$, CCK- 8 reagent $(10 \mu \mathrm{l})$ was diluted with $100 \mu \mathrm{l}$ medium and added to each well. After incubation for 2 hours, the light absorbance at $450 \mathrm{~nm}$ was recorded with a microplate spectrophotometer (K8001, Shanghai Yoke Instrument Co., Ltd., China) (12).

\section{EdU Assay}

The EdU assay was performed using a KFluor488 Click It 5ethynyl-2'-deoxyuridine Imaging Test Kit (KGA331-500; keyGen, Nanjing, China). At 24 hours after transfection, $10^{5}$ cells were seeded in each well of a 24-well plate. After incubation for 24 hours, EdU was added (final concentration of $50 \mu \mathrm{M}$ ). After 2 hours incubation with EdU, cells were fixed with $150 \mu \mathrm{l}$ of $4 \%$ formaldehyde in PBS for $20 \mathrm{~min}$. A 495-nm laser was used to excite kFluor488-azide and images were captured under a fluorescence microscope (IX81, Olympus Corporation, Beijing, China). Nuclei were counterstained with DAPI. 


\section{Western Blotting}

Forty-eight hours post transfection, cells were lysed in RIPA lysis buffer (Merck Group, Germany) for $30 \mathrm{~min}$ on ice. Protein concentrations were determined using a BCA assay kit (Solarbio, China). Lysates with equal amounts were loaded and separated by $12 \%$ SDS-polyacrylamide gel electrophoresis. Proteins were transferred onto polyvinylidene difluoride membranes, followed by probing with target antibodies. Primary antibodies included: Beta-actin (Affinity T0022, 1:1000), and p53 (Affinity AF0879, 1:1000), and the secondary antibody (Affinity S0001, 1:5000) were obtained from Xiangtai Biological Technology Co., Ltd. (China); and ANGPTL4 (Boster A01147, 1:1000) was purchased from Boster Biological Technology (Pleasanton, CA).

\section{Establishment of Mouse Xenograft Tumor Model}

The animal protocol was approved by the Ethical Committee of the Second Affiliated Hospital of Harbin Medical University (a tertiary hospital in northeast China). Male BALB/c nude mice were purchased from Beijing Vital River Laboratory Animal Technology Co., Ltd., and housed in a temperature-controlled, specific-pathogen-free animal facility, with a $12 \mathrm{~h}$ light/12h dark cycle and free access to food and water. Animals were properly treated in accordance with the national and institutional ethical requirements of experimental animals. $1.5 \times 10^{6}$ of HepG2-LNC$\mathrm{KD}$ or HepG2-LNC-NC cells were resuspended in $0.1 \mathrm{ml}$ sterile PBS, and subcutaneously injected in the left flank of mice at the age of 8 weeks. The size of tumor was monitored every morning (length $\mathrm{x}$ width $\mathrm{x}$ depth in $\mathrm{mm}^{3}$ ). Mice were sacrificed 2 weeks after cell injection ( $n=7$ per group).

\section{Immunohistochemistry}

Sections $(10 \mu \mathrm{m})$ of paraffin-embedded xenograft tissue samples were used for immunohistochemistry staining. Slides were incubated with PCNA antibody (1:100 dilution in PBS; AF0239, Affinity Biosciences LTD.) or p53 (1:100 in PBS; Affinity AF0879) at overnight $4^{\circ} \mathrm{C}$. After gentle rinsing off primary antibody solutions, slides were incubated with the secondary antibody (1:200 dilution in PBS; S0001, Affinity Biosciences LTD.) at $37^{\circ} \mathrm{C}$ for 1 hour. Finally, nuclei were counterstained with hematoxylin.

\section{Statistics}

Except for bioinformatics, statistical analyses were done with SPSS version 24.0 (Armonk, NY: IBM Corp.). Continuous data were shown as mean \pm SD. Differences between the two groups were analyzed by independent Student's t-test. p $<0.05$ was considered significant in 2-tailed statistical tests.

\section{RESULTS}

\section{LncRNA AC007639.1 Expression in HCC}

According to the bioinformatic analysis of TCGA-LIHC dataset using the DESeq2 software package, lncRNA AC007639.1 expression in HCC tissues was found to be significantly higher than that of adjacent non-tumor liver tissue (Figures 1A, B). The median level of lncRNA AC007639.1 was used as a cut-off to differentiate the high- and low- AC007639.1 expression groups. Using the GEPIA online tool (13) and R language survival analysis, the patients in the high AC007639.1 expression group were found to have a shorter survival period (Figures 1C, D). These results indicated that AC007639.1 was overexpressed in HCC tissues and the high level of AC007639.1 was indicative of poor prognoses.

\section{Prediction of the Functions of LncRNA AC007639.1 in HCC}

Differentially expressed genes in the high- and low- AC007639.1 expression groups in the TCGA-LIHC dataset were analyzed by DEseq2 (Figure 2A). The enrichment of genes was analyzed using GSVA. The AC007639.1 high expression group was found to have highly enriched HCC up-regulating gene sets (Figure 2B). GO enrichment analysis (14) showed that AC007639.1 was involved in diverse cell functions such as immune functions (Figures 2C, D). LncRNA AC007639.1 was found to regulate the cell cycle (Figure 3A), increase HCC resistance to Doxorubicin (Figure 3B), and decrease the protein expression of p53 (Figure 3C).

\section{LncRNA AC007639.1 in the Proliferation of HCC Cells}

In order to investigate the function of AC007639.1 in HCC, three specific siRNAs were designed to knock down its expression in HCC cell lines, among which both siRNA1 and siRNA3 significantly downregulated AC007639.1 in HepG2, Hep3B, and SMMC-7721 cells ( $\mathrm{p}<0.001$ for both siRNAs, Figure 4A). The lncRNA AC007639.1 knockdown cells showed higher inhibition of proliferation after treated with different concentrations of Doxorubicin than that of the control groups (Figure 4B), which was also confirmed by EdU analysis (Figure 4C). Expression of p53 protein in HepG2, Нep3B, and SMMC-7721 cells were increased when treated with DOX after siRNA1 and siRNA3 transfection (Figure 4D). The above findings suggested that knocking down of AC007639.1 inhibited HCC cell growth, and promoted HCC apoptosis.

\section{LncRNA AC007639.1 in Nude Mice Xenografts}

To explore the role of AC007639.1 in vivo, we developed the HepG2-LNC-KD cells that carried a stable knockdown level of AC007639.1 (confirmed by qRT-PCR, data not shown), and used the cells to create a xenograft tumor mouse model in nude mice. The size of xenograft tumor was significantly smaller in the stable knockdown group at 2 weeks compared with the knockdown group (Figures 5A, B), with a slower time-course growth rate (Figure 5C). The expression of AC007639.1 in the xenograft were significantly lower in the knockdown group, with a significant higher P53 expression (Figure 5D). P53 and PCNA immunohistochemistry showed increased p53-positive cells, 
A

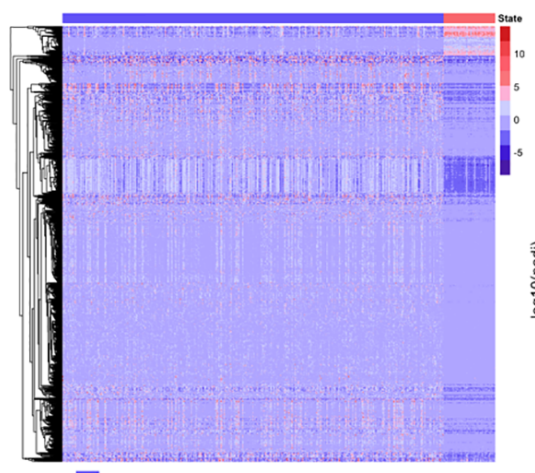

Primary Solid Tumor

B

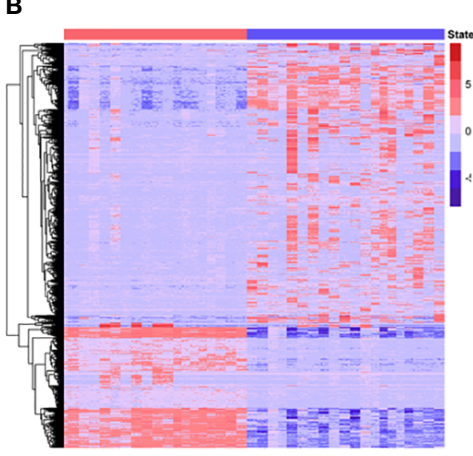

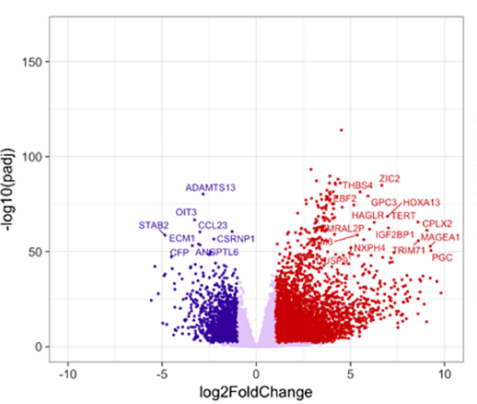

DOWN
NOT
UP

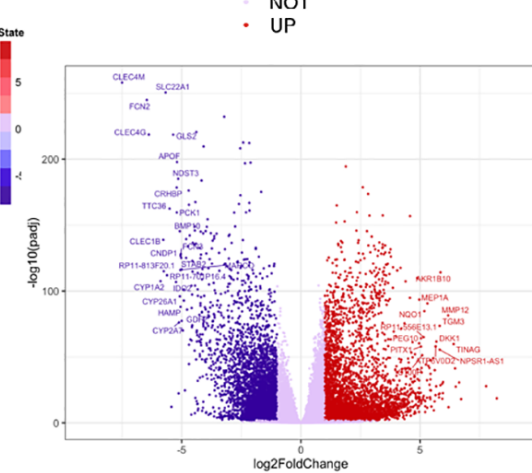

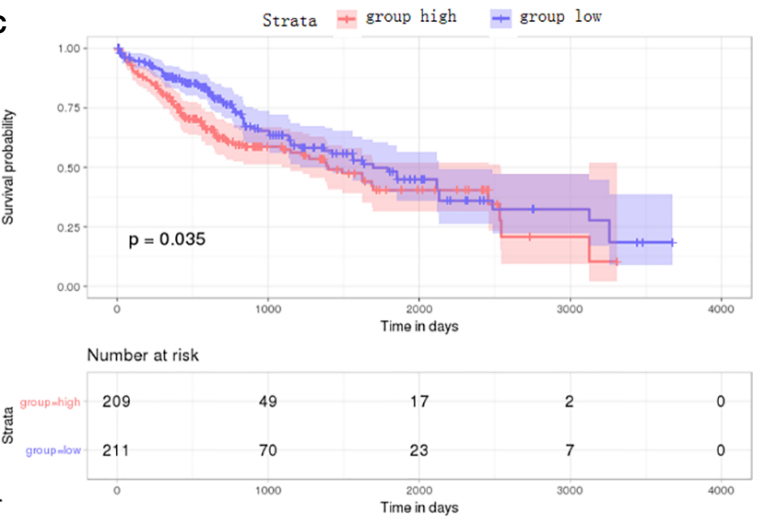

umber of censoring

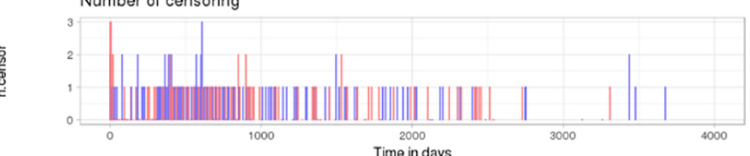

Time in days

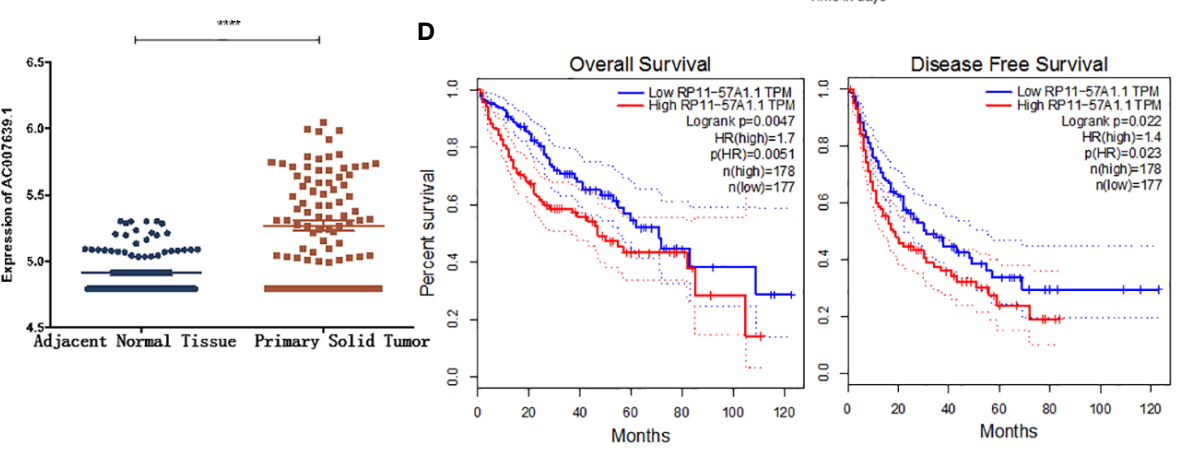

FIGURE 1 | LncRNA AC007639.1 is highly expressed in hepatocellular carcinoma tissues and implies a poor prognosis. (A) Heatmap of transcriptome (left) and volcano plot (middle) of different genes, and IncRNA AC007639.1 expressions in TCGA-LIHC cases (right, n=420, https://gdc.cancer.gov/resources-tcga-users/tcga-code-tables/data-levels); (B) Heatmap of transcriptome (left) and volcano plot (middle) of different genes, and IncRNA AC007639.1 expressions in SRP069212 cases (right, n=355); (C, D) Kaplan-Meier survival curves of TCGA-LIHC dataset analyzed by TCGAbiolinks or SRP069212 dataset analyzed by GEPIA, respectively. ${ }^{* \star \star *} \mathrm{p}<0.001$ (Mann-Whitney test). 


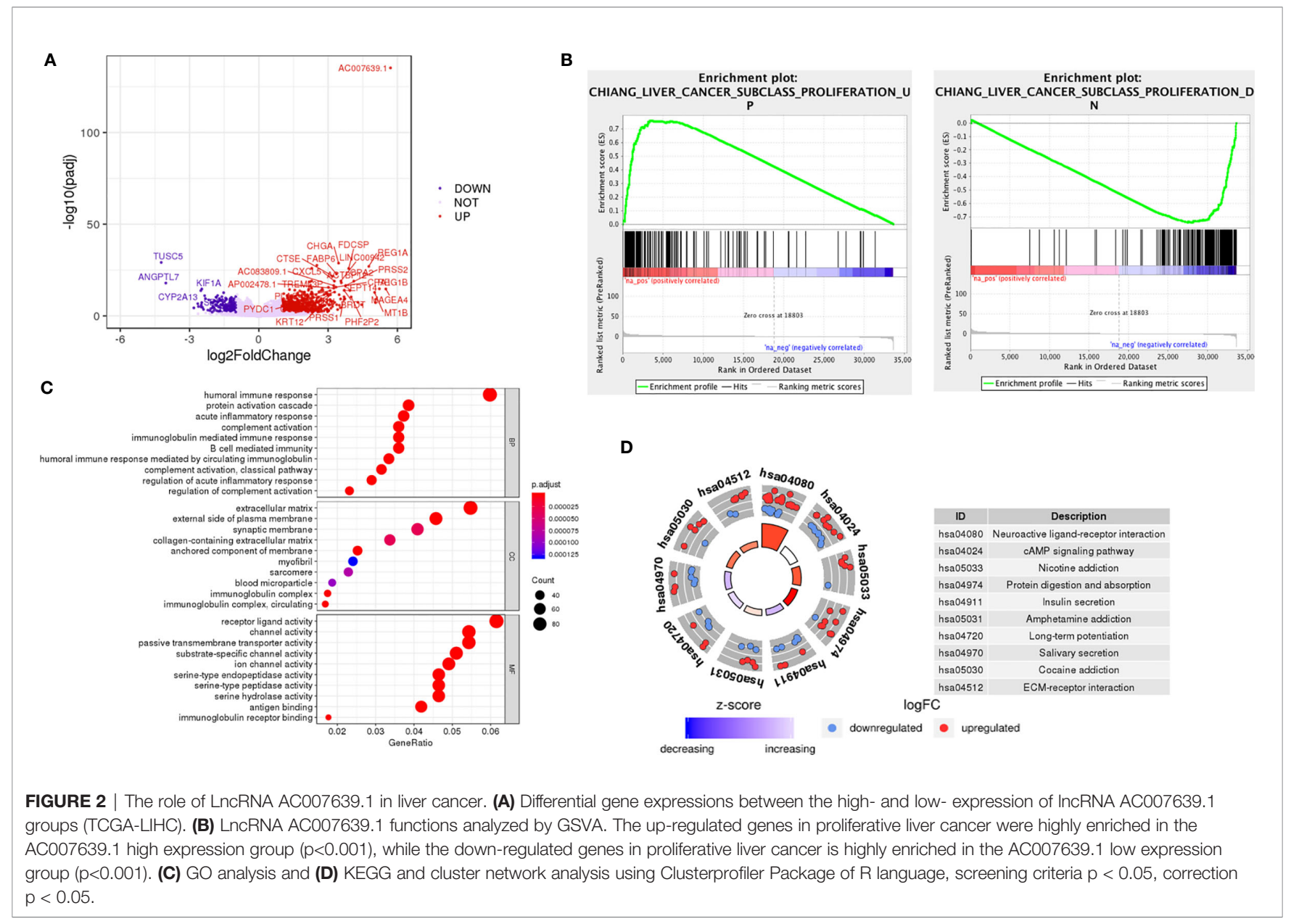

A

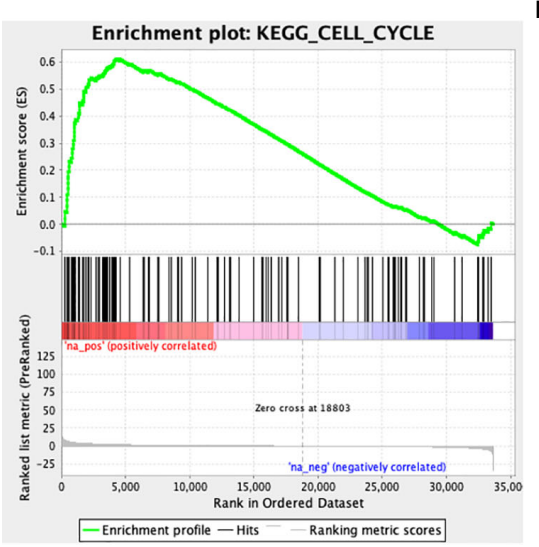

B

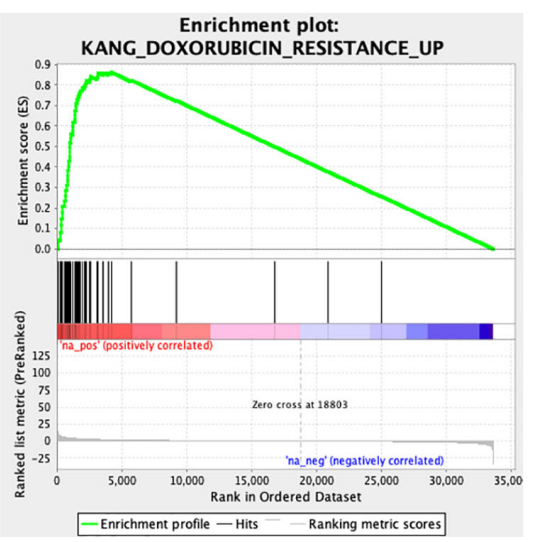

C

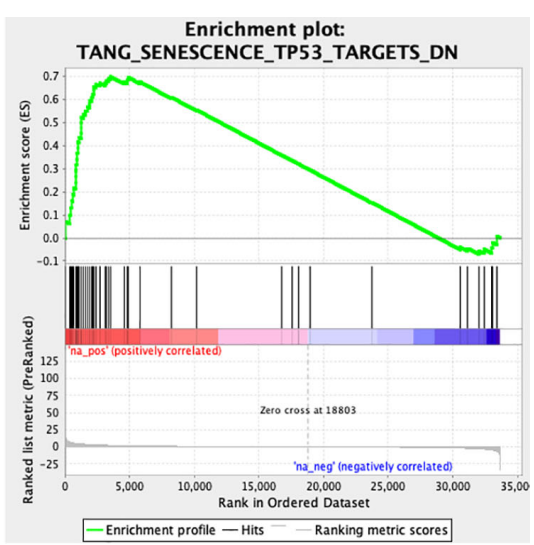

FIGURE 3 | LncRNA AC007639.1 regulates the susceptibility of liver cancer to doxorubicin by inhibiting the p53 pathway, analyzed by GSVA. (A) The effect of IncRNA AC007639.1 on cell cycle ( $p<0.001)$; (B) The effects of IncRNA AC007639.1 on doxorubicin resistance $(p<0.001)$; (C) The effects of IncRNA AC007639.1 on $p 53$ target genes $(p<0.001)$. 
A

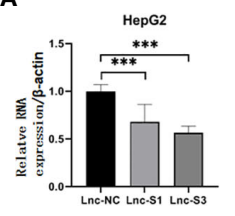

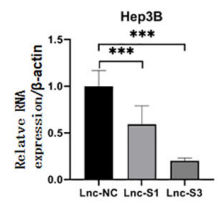

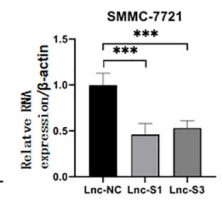

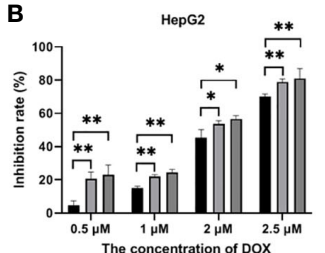

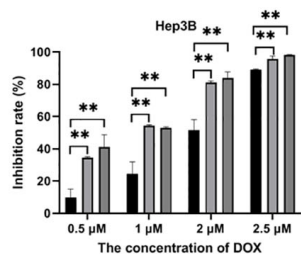

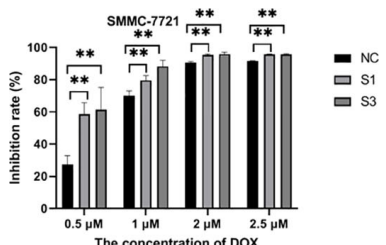

C

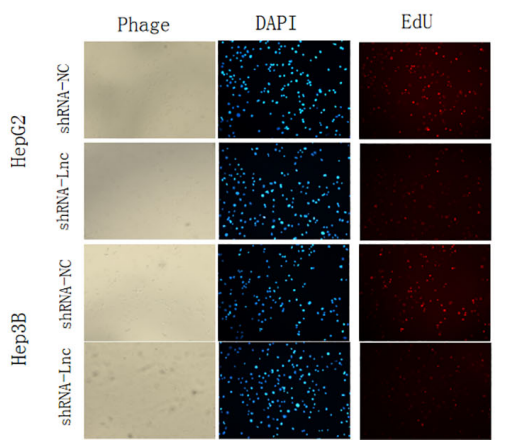

D

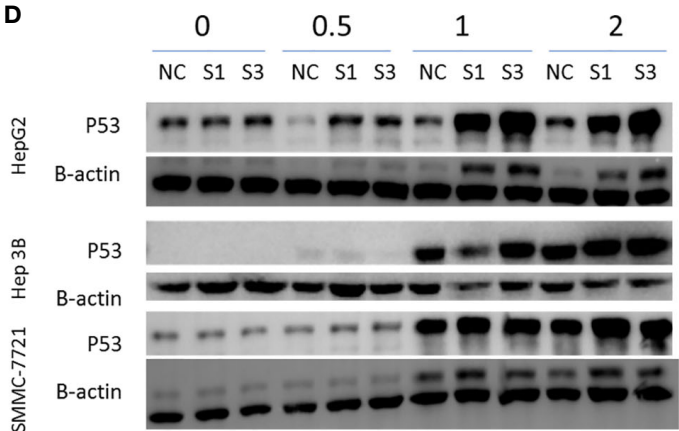

(Dox, $\mu \mathrm{M})$

FIGURE 4 | Knockdown of IncRNA AC007639.1 inhibits the growth of hepatocellular carcinoma cells and promotes apoptosis. (A) qRT-PCR on the relative AC007639.1 expression in HepG2, Hep3B, and SMMC-7721 cells transfected with two IncRNA AC007639.1 or control siRNAs (Lnc-S1, Lnc-S3, or Lnc-NC, respectively); (B) CCK-8 assay showing inhibition of HepG2, Hep3B, and SMMC-7721 cells at different concentrations of doxorubicin after siRNAs transfection; (C) EdU analysis showing cell proliferation after transfection of scrambled shRNA (NC) or shRNA for IncRNA (shRNA-Lnc) (field of view: 200x) in HepG2 or Hep3B cells treated with $2.5 \mu \mathrm{M}$ doxorubicin for 48 hours; (D) Western blots of p53 in the three cells lines after exposure to different concentrations of doxorubicin. NC: scrambled control siRNA or shRNA. S1, siRNA1; S3, siRNA3. ${ }^{*} \mathrm{p}<0.05,{ }^{* *} \mathrm{p}<0.01,{ }^{* * *} \mathrm{p}<0.001$.

A

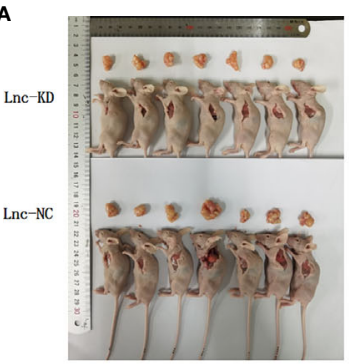

D

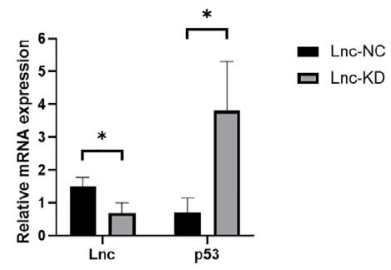

B

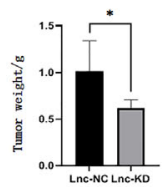

E

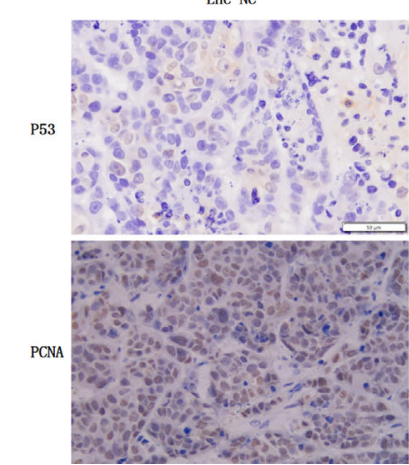

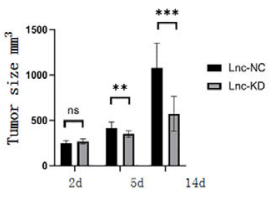

Lnc-KD

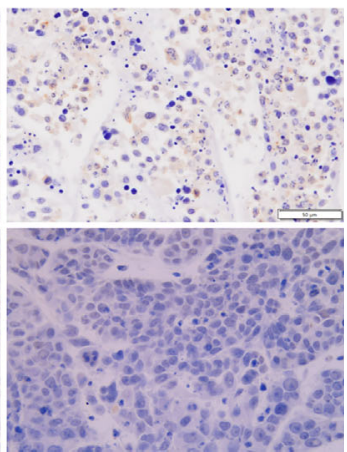

FIGURE 5 | Knockdown of IncRNA AC007639.1 inhibits the growth of tumor xenografts in nude mice. (A) The size of HepG2 xenografts using HepG2-LNC-KD or HepG2-LNC-NC cells at 14 days; (B) Weight of xenografts at collection; (C) Xenografts growth monitored in $\mathrm{mm}^{3}$; (D) Relative AC007639.1 and p53 expressions (normalized to $\beta$-actin) in xenografts with HepG2-LNC-KD or HepG2-LNC-NC cells; (E) p53 and PCNA immunohistochemistry in xenograft tissues (field of view: 200x). Brown colored cells were positive for P53 or PCNA, respectively. ${ }^{*} \mathrm{p}<0.05,{ }^{* \star} \mathrm{p}<0.01,{ }^{\star \star *} \mathrm{p}<0.001$; ns, not statistically significant. 
reduced cell atypia, and reduced PCNA-positive cells after AC007639.1 was knocked down (Figure 5E). These results showed that IncRNA AC007639.1 is important in xenograft tumor growth.

\section{The Mechanism of AC007639.1 in Regulating Cell Functions}

The above bioinformatics analysis indicated that AC007639.1 regulated HCC cell proliferation. We further analyzed the role of IncRNA AC007639.1 by sequencing gene expressions in HepG2 (Figures 6A-C) cells after the transfection of siRNA3. Among the genes with significant changes, ANGPTL4 was selected for further analysis. ANGPTL4 protein was significantly lower in HepG2 cells after AC007639.1 was knocked down (Figure 7A), and ANGPTL4 protein level also decreased in xenografts injected with HepG2-LNC-KD (Figures 7B, C). Knocking down of ANGPTL4 increased the inhibitory effect of Doxorubicin in HepG2, Hep3B, and SMMC7721 cells (Figures 7D, E).

\section{DISCUSSION}

LncRNAs and endonuclease (15) are involved in the pathogenesis of liver cancer. Most lncRNAs promote the proliferation of HCC through microRNAs (16).
A

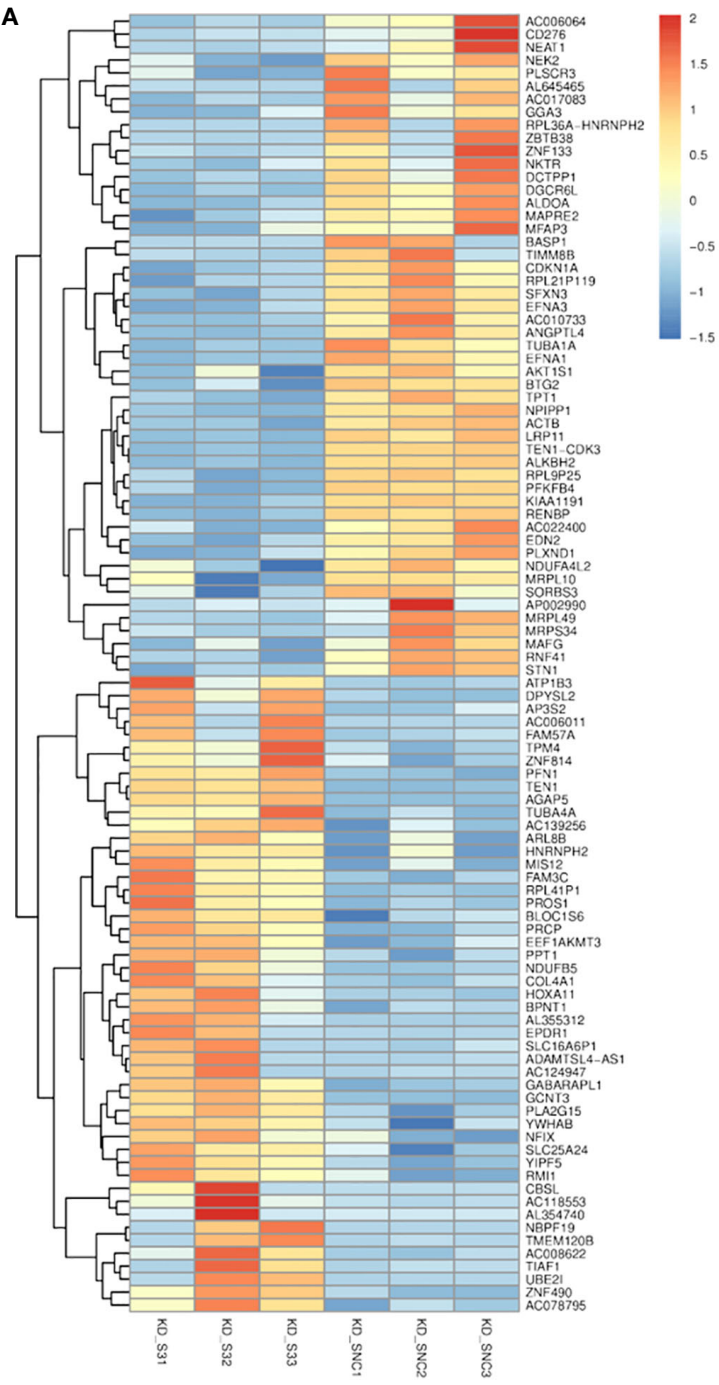

B

\section{KD_S3VSKD_SNC}

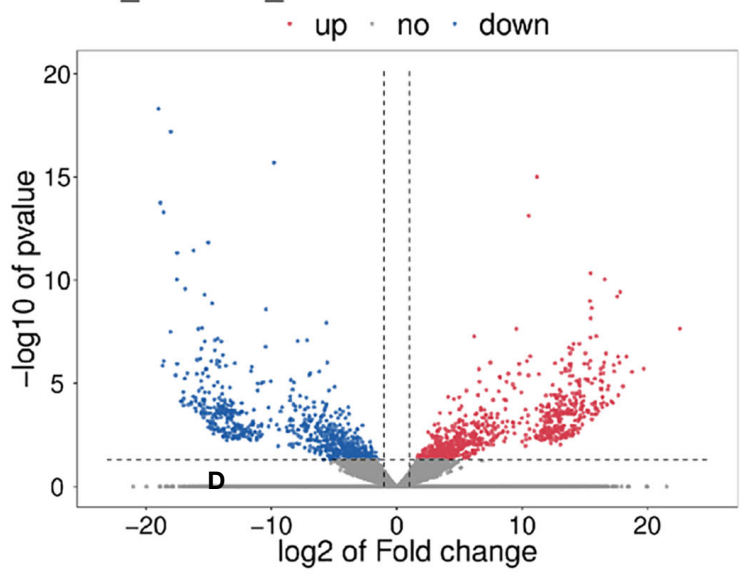

C

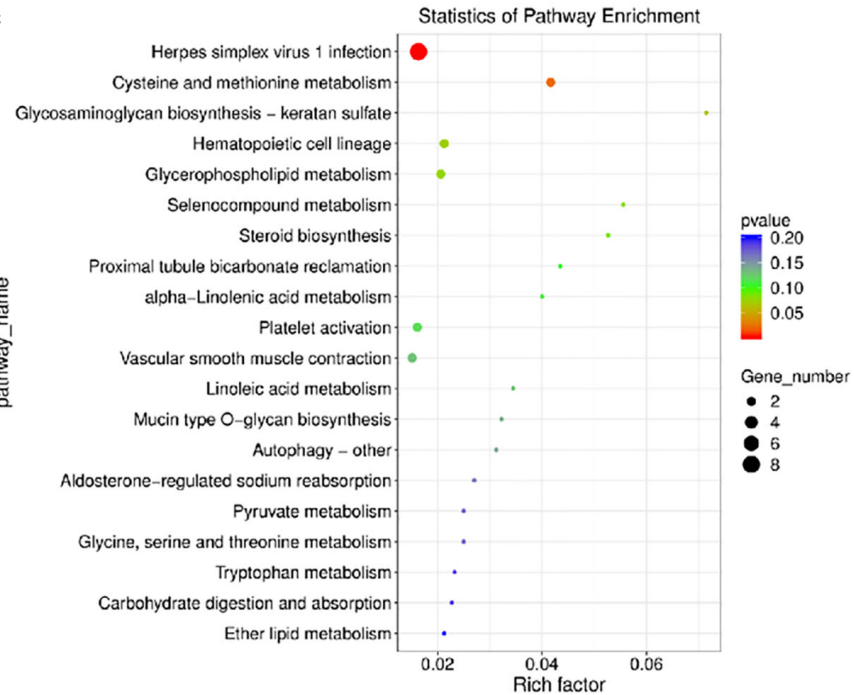

FIGURE 6 | The involvement of IncRNA AC007639.1 in different pathways. (A) Heatmap showing different gene expressions after the knock down of IncRNA AC007639.1 in HepG2 cells using siRNA3; (B) Volcano plot of different gene expressions after the knock down of IncRNA AC007639.1 in HepG2 cell line; (C) Pathways enrichment after the knock down of IncRNA AC007639.1 in HepG2 cell line. 


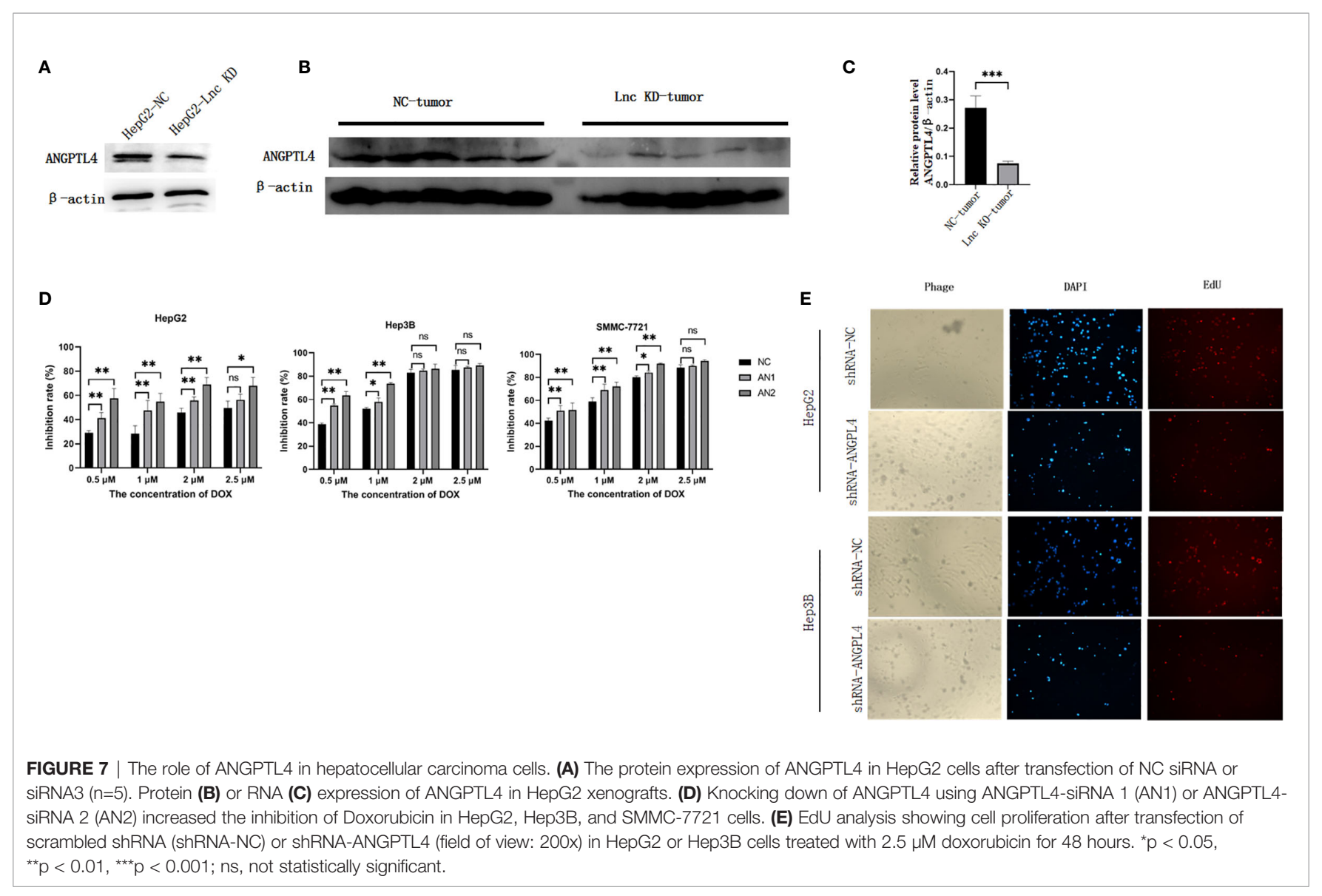

The application of bioinformatic analyses facilitated the identification of candidate lncRNAs and related signaling pathways for indispensable mechanistic studies. According to the findings from the TCGA-LIHC dataset, lncRNA AC007639.1 was knocked down in three HCC cell lines using specific siRNAs or shRNA, with the finding of significantly more cell death and inhibition of cell proliferation, which showed lncRNA AC007639.1 is involved in HCC pathogenesis.

We tested the role of IncRNA AC007639.1 in three cell lines of hepatic origin, HepG2, Hep3B, and SMMC-7721. HepG2 is characterized by hepatitis $\mathrm{B}$ virus negative and non-tumorigenic, while Hep3B is characterized by hepatitis B virus positive and tumorigenic (17), and SMMC-7721 has been suspected of its liver origin due to contamination concerns (18). Therefore, to avoid controversies, SMMC-7721 cells were just used to test the efficacy of siRNA knock down (19), but not selected for further mechanistic experiments. Doxorubicin is among the most used chemotherapeutics against human cancers (20), the resistance of which is related with lncRNA AC007639.1 as shown in this study.

ANGPTL4 protein belongs to the angiopoietin (ANG)-related family. It is highly expressed in numerous organs including liver, and is be stimulated by inflammatory or hypoxic conditions (21, 22). However, the roles of ANGPTL4 in human cancers are controversial in different experimental models and proposed pathways. Overexpression of ANGPTL4 promotes tumorigenesis and metastasis (23), whereas it presents anti-metastatic activity through inhibition of vascular permeability and invasiveness (24). In the clinical settings, a high serum ANGPTL4 protein level in HCC patients is predictive of liver cirrhosis and intrahepatic metastasis (25). But levels of ANGPTL4 protein in tumor tissues are significantly lower than that in non-tumor tissues of the same HCC patients (26). Knockdown of ANGPTL4 inhibits the development of human gastric cancer (27). Our findings showed that the expression of ANGPTL4 is controlled by lncRNA AC007639.1, and ANGPTL4 contributed to the resistance of HCC cells to doxorubicin.

Taken together, we showed by bioinformatics and mechanistic studies that lncRNA AC007639.1 was involved in the pathogenesis of HCC by decreasing apoptosis and increasing resistance to chemotherapy. LncRNA AC007639.1 could be a valuable prognostic predictor as well as treatment target in HCC patients.

\section{DATA AVAILABILITY STATEMENT}

The datasets presented in this study can be found in online repositories. The names of the repository/repositories and 
accession number(s) can be found below: (https://www.ncbi.nlm. nih.gov/genbank/), PRJNA716423.

\section{ETHICS STATEMENT}

The animal study was reviewed and approved by Ethical Committee of Second Affiliated Hospital of Harbin Medical University.

\section{AUTHOR CONTRIBUTIONS}

YY and YB conceived the study, analyzed and interpreted patient data, and were major contributors in writing the

\section{REFERENCES}

1. The American Cancer Society medical and editorial content team. Key Statistics About Liver Cancer (2021). Available at: https://www.cancer.org/cancer/livercancer/about/what-is-key-statistics.html (Accessed March 2, 2020).

2. Li XM, Ma L, Yang YB, Shi ZJ, Zhou SS. Analyses of Prognostic Indices of Chronic Liver Failure Caused by Hepatitis Virus. World J Gastroenterol (2005) 11(18):2841-3. doi: 10.3748/wjg.v11.i18.2841

3. Yang Y, Deng L, Li X, Shi Z, Chen D, Chen X, et al. Evaluation of the Prognosis of Fulminant Viral Hepatitis in Late Pregnancy by the MELD Scoring System. Eur J Clin Microbiol Infect Dis (2012) 31(10):2673-8. doi: 10.1007/s10096-012-1613-y

4. Yang Y, Deng L, Li X, Shi Z, Jiang P, Chen D, et al. Analysis of PrognosisAssociated Factors in Fulminant Viral Hepatitis During Pregnancy in China. Int J Gynaecol Obstet (2011) 114(3):242-5. doi: 10.1016/j.ijgo.2011.03.017

5. Deng L, Li X, Shi Z, Jiang P, Chen D, Ma L. Maternal and Perinatal Outcome in Cases of Fulminant Viral Hepatitis in Late Pregnancy. Int $J$ Gynecol Obstet (2012) 119(2):145-8. doi: 10.1016/j.ijgo.2012.05.041

6. Ma L, Alla N, Li X, Mynbaev O, Shi Z. Mother to Child Transmission of HBV: Review of Current Clinical Management and Prevention Strategies. Rev Med Virol (2014) 24(6):396-406. doi: 10.1002/rmv.1801

7. Engreitz JM, Haines JE, Perez EM, Munson G, Chen J, Kane M, et al. Local Regulation of Gene Expression by Lncrna Promoters, Transcription and Splicing. Nature (2016) 539(7629):452-5. doi: 10.1038/nature20149

8. Lin X, Xiang X, Feng B, Zhou H, Wang T, Chu X, et al. Targeting Long nonCoding Rnas in Hepatocellular Carcinoma: Progress and Prospects. Front Oncol (2021) 11:670838. doi: 10.3389/fonc.2021.670838

9. Naipauer J, García Solá ME, Salyakina D, Rosario S, Williams S, Coso O, et al. A non-Coding RNA Network Involved in KSHV Tumorigenesis. Front Oncol (2021) 11:687629. doi: 10.3389/fonc.2021.687629

10. Huang J, Zheng Y, Xiao X, Liu C, Lin J, Zheng S, et al. A Circulating Long Noncoding RNA Panel Serves as a Diagnostic Marker for Hepatocellular Carcinoma. Dis Markers (2020) 2020:5417598. doi: 10.1155/2020/5417598

11. Love MI, Huber W, Anders S. Moderated Estimation of Fold Change and Dispersion for RNA-Seq Data With Deseq2. Genome Biol (2014) 15(12):550. doi: 10.1186/s13059-014-0550-8

12. Yuan Q, Gao C, Lai XD, Chen LY, Lai TB. Analysis of Long Noncoding RNA ZNF667-AS1 as a Potential Biomarker for Diagnosis and Prognosis of Glioma Patients. Dis Markers (2020) 2020:8895968. doi: 10.1155/2020/8895968

13. Tang Z, Li C, Kang B, Gao G, Li C, Zhang Z. GEPIA: A Web Server for Cancer and Normal Gene Expression Profiling and Interactive Analyses. Nucleic Acids Res (2017) 45(W1):W98-102. doi: 10.1093/nar/gkx247

14. The Gene Ontology Consortium. The Gene Ontology Resource: 20 Years and Still Going Strong. Nucleic Acids Res (2019) 47(D1):D330-8. doi: 10.1093/nar/ gky1055

15. Zhang Y, Liu X, Liu L, Chen J, Hu Q, Shen S, et al. Upregulation of FEN1 Is Associated With the Tumor Progression and Prognosis of Hepatocellular Carcinoma. Dis Markers (2020) 2020:2514090. doi: 10.1155/2020/2514090 manuscript. YB, MD, DL, YL, SY, LW, HL, GC, XL, and XS obtained experiment data. All authors contributed to the article and approved the submitted version.

\section{SUPPLEMENTARY MATERIAL}

The Supplementary Material for this article can be found online at: https://www.frontiersin.org/articles/10.3389/fonc.2021. 715541/full\#supplementary-material

Supplementary Figure 1 | The map of shRNA vector.

Supplementary Table 1 | Primers for qRT-PCR and siRNAs.

16. Xiong D, He R, Dang Y, Wu H, Feng Z, Chen G. The Latest Overview of Circrna in the Progression, Diagnosis, Prognosis, Treatment, and Drug Resistance of Hepatocellular Carcinoma. Front Oncol (2020) 10:608257. doi: 10.3389/ fonc.2020.608257

17. Qiu GH, Xie X, Xu F, Shi X, Wang Y, Deng L. Distinctive Pharmacological Differences Between Liver Cancer Cell Lines Hepg2 and Hep3B. Cytotechnology (2015) 67(1):1-12. doi: 10.1007/s10616-014-9761-9

18. Rebouissou S, Zucman-Rossi J, Moreau R, Qiu Z, Hui L. Note of Caution: Contaminations of Hepatocellular Cell Lines. J Hepatol (2017) 67(5):896-7. doi: 10.1016/j.jhep.2017.08.002

19. Shi Z, Nicholson RH, Jaggi R, Nicholson AW. Characterization of Aquifex Aeolicus Ribonuclease III and the Reactivity Epitopes of its Pre-Ribosomal RNA Substrates. Nucleic Acids Res (2011) 39(7):2756-68. doi: 10.1093/nar/gkq1030

20. Xu B, Yuan L, Hu Y, Xu Z, Qin JJ, Cheng XD. Synthesis, Characterization, Cellular Uptake, and In Vitro Anticancer Activity of Fullerenol-Doxorubicin Conjugates. Front Pharmacol (2020) 11:598155. doi: 10.3389/fphar.2020.598155

21. Ng KT, Xu A, Cheng Q, Guo DY, Lim ZX, Sun CK, et al. Clinical Relevance and Therapeutic Potential of Angiopoietin-Like Protein 4 in Hepatocellular Carcinoma. Mol Cancer (2014) 13:196. doi: 10.1186/1476-4598-13-196

22. Zhu P, Goh YY, Chin HF, Kersten S, Tan NS. Angiopoietin-Like 4: A Decade of Research. Biosci Rep (2012) 32(3):211-9. doi: 10.1042/BSR20110102

23. Kim SH, Park YY, Kim SW, Lee JS, Wang D, DuBois RN. ANGPTL4 Induction by Prostaglandin E2 Under Hypoxic Conditions Promotes Colorectal Cancer Progression. Cancer Res (2011) 71(22):7010-20. doi: 10.1158/0008-5472.CAN-11-1262

24. Galaup A, Cazes A, Le Jan S, Philippe J, Connault E, Le Coz E, et al. Angiopoietin-Like 4 Prevents Metastasis Through Inhibition of Vascular Permeability and Tumor Cell Motility and Invasiveness. Proc Natl Acad Sci USA (2006) 103(49):18721-6. doi: 10.1073/pnas.0609025103

25. Li H, Ge C, Zhao F, Yan M, Hu C, Jia D, et al. Hypoxia-Inducible Factor 1 AlphaActivated Angiopoietin-Like Protein 4 Contributes to Tumor Metastasis via Vascular Cell Adhesion Molecule-1/Integrin Beta1 Signaling in Human Hepatocellular Carcinoma. Hepatology (2011) 54(3):910-9. doi: 10.1002/hep.24479

26. Zhang H, Wei S, Ning S, Jie Y, Ru Y, Gu Y. Evaluation of Tgfbeta, XPO4, Elf5a2 and ANGPTL4 as Biomarkers in HCC. Exp Ther Med (2013) 5(1):11927. doi: 10.3892/etm.2012.750

27. Chen JW, Luo YJ, Yang ZF, Wen LQ, Huang L. Knockdown of AngiopoietinLike 4 Inhibits the Development of Human Gastric Cancer. Oncol Rep (2018) 39(4):1739-46. doi: 10.3892/or.2018.6253

Conflict of Interest: Author SY was employed by the company "Harbin Nachuan Bio-Science Technology Co., LTD.”.

The remaining authors declare that the research was conducted in the absence of any commercial or financial relationships that could be construed as a potential conflict of interest.

Publisher's Note: All claims expressed in this article are solely those of the authors and do not necessarily represent those of their affiliated organizations, or those of the publisher, the editors and the reviewers. Any product that may be evaluated in 
this article, or claim that may be made by its manufacturer, is not guaranteed or endorsed by the publisher.

Copyright $\odot 2021$ Bai, Ding, Lu, Li, Yao, Wang, Li, Cui, Li, Sun and Yang. This is an open-access article distributed under the terms of the Creative Commons Attribution
License (CC BY). The use, distribution or reproduction in other forums is permitted, provided the original author(s) and the copyright owner(s) are credited and that the original publication in this journal is cited, in accordance with accepted academic practice. No use, distribution or reproduction is permitted which does not comply with these terms. 